\title{
A new crystalline phase of the electroluminescent material tris(8- hydroxyquinoline) aluminum exhibiting blueshifted fluorescence
}

Cite as: J. Chem. Phys. 114, 9625 (2001); https://doi.org/10.1063/1.1369157

Submitted: 30 October 2000 . Accepted: 12 March 2001. Published Online: 21 May 2001

M. Braun, J. Gmeiner, M. Tzolov, M. Coelle, F. D. Meyer, W. Milius, H. Hillebrecht, O. Wendland, J. U. von Schütz, and W. Brütting

\section{ARTICLES YOU MAY BE INTERESTED IN}

Organic electroluminescent diodes

Applied Physics Letters 51, 913 (1987); https://doi.org/10.1063/1.98799

Electroluminescence of doped organic thin films

Journal of Applied Physics 65, 3610 (1989); https://doi.org/10.1063/1.343409

Relationship between electroluminescence and current transport in organic heterojunction light-emitting devices

Journal of Applied Physics 79, 7991 (1996); https://doi.org/10.1063/1.362350

\section{Lock-in Amplifiers up to $600 \mathrm{MHz}$}
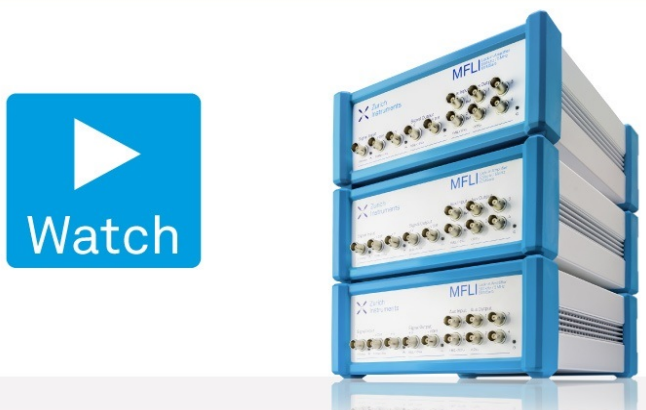


\title{
A new crystalline phase of the electroluminescent material tris(8-hydroxyquinoline) aluminum exhibiting blueshifted fluorescence
}

\author{
M. Braun ${ }^{\mathrm{a})}$ \\ 3. Institute of Physics, University of Stuttgart, 70550 Stuttgart, Germany \\ J. Gmeiner, M. Tzolov, and M. Coelle \\ Experimental Physics II, University of Bayreuth, 95440 Bayreuth, Germany \\ F. D. Meyer, W. Milius, and H. Hillebrecht \\ Inorganic Chemistry I, University of Bayreuth, 95440 Bayreuth, Germany \\ O. Wendland and J. U. von Schütz \\ 3. Institute of Physics, University of Stuttgart, 70550 Stuttgart, Germany \\ W. Brütting ${ }^{\text {b) }}$ \\ Experimental Physics II, University of Bayreuth, 95440 Bayreuth, Germany
}

(Received 30 October 2000; accepted 12 March 2001)

\begin{abstract}
We report on two different crystalline phases of tris(8-hydroxyquinoline) aluminum $\left(\mathrm{Alq}_{3}\right)$ which were obtained by thermal sublimation in a horizontal glass tube. These phases are investigated by x-ray powder diffraction, Raman and infrared spectroscopy, and low temperature photoluminescence measurements. Apart from the already known $\alpha$ phase we could identify a new crystalline phase of $\mathrm{Alq}_{3}\left(\delta-\mathrm{Alq}_{3}\right.$ ) showing blueshifted fluorescence. As compared to the $\alpha$ phase this new phase is characterized by a larger unit cell volume, a reduced number of Raman lines in the energy range between 70 and $700 \mathrm{~cm}^{-1}$, a blueshift of the photoluminescence maximum by about $0.2 \mathrm{eV}$, and a decreased intersystem crossing to the triplet state. These differences are interpreted in terms of the isomery of the $\mathrm{Alq}_{3}$ molecule. It is supposed that the new phase contains the facial isomer, whereas in the other phases only the meridianal isomer was reported. Low temperature photoluminescence spectra show a well-resolved vibronic progression with about the same spacing of $550 \mathrm{~cm}^{-1}$ for both crystalline phases of $\mathrm{Alq}_{3}$. Site-selective photoluminescence measurements reveal the existence of an additional redshifted featureless emission, which is ascribed to energy relaxation into low-lying states. (C) 2001 American Institute of Physics.
\end{abstract}

[DOI: 10.1063/1.1369157]

\section{INTRODUCTION}

8-hydroxyquinoline metal chelate complexes were used for many years in analytical chemistry for a gravimetric determination of various metal cations in solution. ${ }^{1,2}$ The development of more convenient spectroscopic techniques has meanwhile replaced this method and concomitantly decreased the interest of researching chemists in this reagent. However, the report of efficient electroluminescent devices based on tris(8-hydroxyquinoline) aluminum $\left(\mathrm{Alq}_{3}\right)$ attracted renewed interest in this class of materials for use as active medium in organic light-emitting diodes (OLEDs). ${ }^{3}$ After more than 10 years of intense research and development of OLEDs $\mathrm{Alq}_{3}$ still continues to be the workhorse in low-molecular weight materials for OLEDs. Apart from its use as an electron transporting layer in a heterolayer device in combination with a hole transporting material like an aromatic amine, where green light emission is generated by electron-hole recombination in $\mathrm{Alq}_{3}$, it also serves as host

\footnotetext{
a) Present address: School of Chemistry and Biochemistry, Georgia Institute of Technology, Atlanta.

b) Author to whom correspondence should be addressed. Electronic mail: Wolfgang.Bruetting@uni-bayreuth.de
}

material for various dyes to tune the emission color from green to red. ${ }^{4}$ Many studies in this field have focused on the optimization of the device characteristics with respect to efficiency and long-term stability ${ }^{5-9}$ or to the understanding of the charge transport properties ${ }^{10-13}$ of amorphous thin films. However, so far comparatively few investigations have been devoted to the material's electronic and optical propertiesin particular in the crystalline state-as well as the dependence of these properties on the preparation conditions. Only very recently, a systematic study of the optical properties of solutions, amorphous films, and different polymorphic crystalline phases of $\mathrm{Alq}_{3}$ was published. ${ }^{14}$ Three new crystalline phases $\left(\alpha-, \beta-\right.$, and $\left.\gamma-\mathrm{Alq}_{3}\right)$ were identified. The vibronic structure of the fluorescence was resolved for the first time in these crystals at low temperature and the correlation between the molecular packing and the spectral features of the fluorescence was established.

Another unresolved issue concerns the isomery of the $\mathrm{Alq}_{3}$ molecule. It is well-known that octahedral complexes of the type $\mathrm{MN}_{3} \mathrm{O}_{3}$, where $\mathrm{M}$ is a metal and $\mathrm{N}$ and $\mathrm{O}$ stand for the nitrogen and oxygen atoms in the quinoline ligands, can occur in two different geometrical isomers: meridianal and facial (see Fig. 1). Curioni et al. have calculated the 



FIG. 1. Chemical structure of tris(8-hydroxyquinoline) aluminum $\left(\mathrm{Alq}_{3}\right)$ and schematic drawing of the two geometrical $\mathrm{Alq}_{3}$ isomers (mer: meridianal, fac: facial). The position of the organic ligands is only shown schematically by the gray bars. (For calculated three-dimensional structures of the two isomers see Ref. 15.)

ground state properties of the two isomers from $a b$ initio quantum chemical calculations on isolated $\mathrm{Alq}_{3}$ molecules in the neutral and charged state. ${ }^{15}$ Martin et al. have recently extended these results to the excited state properties of the meridianal isomer, ${ }^{16}$ which is predicted to be thermodynamically more stable than the facial one. Consequently, up to now experimental investigations have given no evidence for the presence of the facial isomer. Therefore, it is generally believed that the meridianal isomer is predominant, both in amorphous films and crystals of $\mathrm{Alq}_{3}$. Nevertheless, early investigations by mass spectroscopy clearly indicated the existence of two isomers of $\mathrm{Alq}_{3} .{ }^{17}$

In this paper we report on growth, $\mathrm{x}$-ray powder diffraction and optical characterization of a new crystalline phase of $\mathrm{Alq}_{3}$ exhibiting a strongly blueshifted fluorescence as compared to the other known phases of $\mathrm{Alq}_{3}$. We discuss these results in terms of the isomery of the $\mathrm{Alq}_{3}$ molecule.

\section{EXPERIMENTAL}

The investigations described below were performed on polycrystalline material obtained as different fractions of a horizontal train sublimation. The aluminum tris-chelate com- plex $\left(\mathrm{Alq}_{3}\right.$, chemical structure shown in Fig. 1) used for sublimation was purchased from different suppliers, e.g., Aldrich or Tokyo Kasei. We have also used $\mathrm{Alq}_{3}$ synthesized by ourselves, following a slightly modified synthetic route from Ref. 18. Before describing details of the preparation it is important to note that all complexes from different origin showed the same behavior in the sublimation and also the $\mathrm{x}$-ray and optical characterization gave the same results.

The sublimation was performed in a horizontal glass tube (length $90 \mathrm{~cm}$, width $1 \mathrm{~cm}$ ). At first the $\mathrm{Alq}_{3}$ source material was filled in a smaller glass tube (length $10 \mathrm{~cm}$, width $8 \mathrm{~mm}$ ) under ambient conditions. This tube was put into the larger glass tube with the open side to the bottom of the larger tube. For each sublimation run we used about 1.5 $\mathrm{g}$ of $\mathrm{Alq}_{3}$. The heating of the samples was carried out in a high temperature oven (supplier: GERO, Hochtemperaturöfen, Germany). Prior to heating, the tube was evacuated to 1 $\mathrm{Pa}$ at room temperature for half an hour. Under this vacuum the temperature was increased in three steps over about 4 hours until $400{ }^{\circ} \mathrm{C}$ were reached. At this temperature the sublimation was performed for another 4 hours. After the sublimation was finished we observed three zones with different crystalline material in the glass tube. In the hottest zone of the growth area, at a distance of about $15 \mathrm{~cm}$ from the short tube containing the source material, there was a 1 $\mathrm{cm}$ wide region with very small needlelike crystals with white or slightly yellow appearance, which showed blue fluorescence when excited with UV light (fraction 1). This zone was followed by the main fraction (about $5 \mathrm{~cm}$ ) with yellow cubic crystals with dimensions up to $500 \times 500 \times 500$ $\mu \mathrm{m}^{3}$ showing yellowish-green fluorescence (fraction 2). In the subsequent colder zone of the sublimation tube we obtained another fraction (about $3 \mathrm{~cm}$ ) with dark-yellow needlelike crystals (size $50 \times 50 \times 500 \mu \mathrm{m}^{3}$ ) exhibiting also yellowish-green fluorescence (fraction 3). Finally, in the glass tube containing the source material there remained a small amount of brown flaky material as residue. The temperature gradient along the growth zone was approximately linear between ca. $370{ }^{\circ} \mathrm{C}$ (beginning of fraction 1) and ca. $330^{\circ} \mathrm{C}$ (end of fraction 3 ).

We observed different solubility of the investigated fractions in organic solvents. While fraction 3 and apart from a small residue also fraction 2 were readily dissolved in chloroform at relatively high concentration (more than $1 \%$ by weight), the solubility of fraction 1 was extremely poor. It took several hours to dissolve a sizeable amount in chloroform. Interestingly, the solution showed the same yellowishgreen fluorescence as solutions of fraction 3. We further note that amorphous films prepared from fraction 1 by evaporation onto substrates at room temperature did not show blue fluorescence but the usual yellowish-green emission of $\mathrm{Alq}_{3}$. All these results confirm that in the studied fractions, in particular in fraction 1, the $\mathrm{Alq}_{3}$ complex is not decomposed, but has different crystalline packing and/or molecular geometry.

X-ray powder diffractograms were obtained with Gemonocromated $\mathrm{Cu} K_{\alpha 1}$ radiation $(\lambda=1.54056 \AA)$ on a transmission powder diffractometer in Debye-Scherrer geometry (Siemens D5000). Data were collected at room temperature 


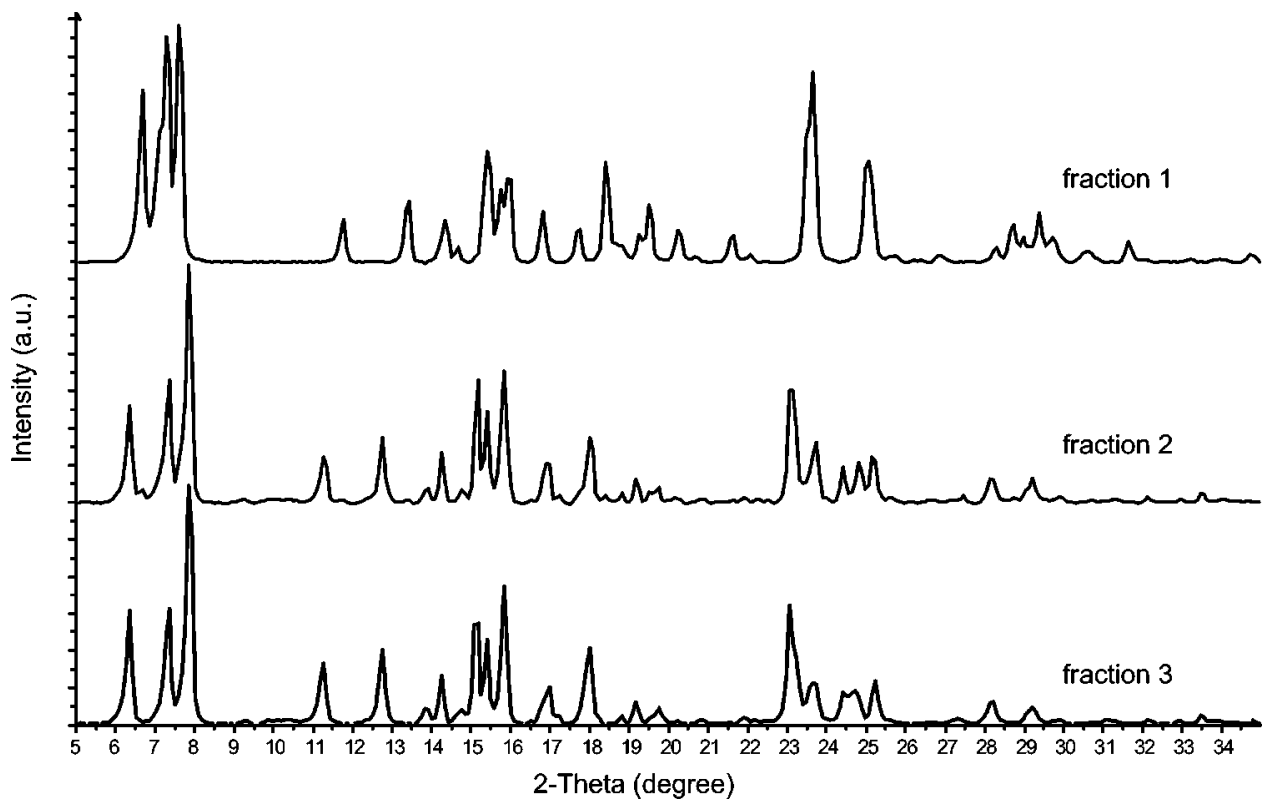

FIG. 2. X-ray powder diffractograms of polycrystalline $\mathrm{Alq}_{3}$ fractions 1,2 , and 3 obtained from sublimation $(2 h$, step width $\Delta 2 \Theta=0.083^{\circ}$ ).

in the $2 \Theta$-range $5^{\circ}-35^{\circ}(\Theta-2 \Theta$ mode, linear PSD). Samples were prepared in capillaries. Data evaluation was done using the DIFFRACT PLUS software (Bruker-AXS).

Photoluminescence spectra were recorded with the sample in a glass cryostat with superfluid helium at $1.3 \mathrm{~K}$. The samples were excited with the $363.8 \mathrm{~nm}$ line of an argon ion laser (Spectra Physics) at a power of $10 \mathrm{~mW}$. The optical emission spectra were recorded with a system of filter, monochromator (Jobin-Yvon) and photomultiplier in a $90^{\circ}$ setup with respect to the optical excitation path. For the measurements at $6 \mathrm{~K}$ the sample was placed in a helium flow cryostat and was excited by the light of an XBO lamp (700 W) passed through a $\mathrm{H}_{2} \mathrm{O}$ filter and a double monochromator (Spex). The emission of the sample was recorded in a $90^{\circ}$ setup with a system of filter, double-monochromator (Spex) and photomultiplier. For the recording of the delayed emission we used two phase-stable $50 \mathrm{~Hz}$ choppers in the excitation and detection path. The excitation and emission spectra, respectively, were corrected by the spectral characteristics of the XBO lamp, the used monochromator and detector system. Infrared and Raman measurements were performed with a Fourier transform spectrometer (Bruker IFS55) at room temperature. The use of a laser emitting at $1064 \mathrm{~nm}$ allowed to perform the Raman measurements on the material as obtained by the sublimation process without any further modification. Double-side polished crystalline $\mathrm{Si}$ wafers were used as substrates for the infrared transmission measurements. The different $\mathrm{Alq}_{3}$ fractions were deposited on these substrates by casting from solution. Because of the poor solubility the blue fluorescence was retained in films of fraction 1 cast from solution. Infrared measurements were also performed on the materials by incorporation into $\mathrm{KBr}$ platelets.

\section{RESULTS}

\section{A. X-ray analysis}

X-ray powder diffractograms were measured from fractions 1, 2, and 3 (Fig. 2). A comparison to the published data ${ }^{14}$ shows, that fraction 3 corresponds to $\alpha$-Alq $\mathrm{Al}_{3}$. According to the cell parameters (see below) fraction 1 represents a new crystalline modification of $\mathrm{Alq}_{3}$. Fraction 2 is a mixture of $\alpha-\mathrm{Alq}_{3}$ and the new modification with $\alpha$ - $\mathrm{Alq}_{3}$ as the main share. This phase analysis by means of x-ray powder diffraction is in agreement with the optical measurements to be discussed below.

The powder diffractogram of fraction 1 with higher resolution shown in Fig. 3 was used for a quantitative analysis. Therein the linewidth is caused experimentally without indications for amorphous by-products. The 25 lowest $2 \Theta$ peaks were used in the indexing routine DICVOL91. ${ }^{19,20}$ The best figure of merit belongs to a triclinic cell with $a$ $=14.501(15) \AA, b=12.651(14) \AA, c=13.402(11) \AA, \alpha$ $=82.74(7)^{\circ}, \quad \beta=114.22(7)^{\circ}, \quad \gamma=93.85(16)^{\circ}, \quad$ and $V$ $=2224(2) \AA^{3}$. With the assumption of four molecules per unit cell a density of $1.372 \mathrm{~g} / \mathrm{cm}^{3}$ is obtained. This value is comparable to $\alpha$ - $\mathrm{Alq}_{3}\left(1.373 \mathrm{~g} / \mathrm{cm}^{3}\right) .{ }^{14}$ Table I gives the observed and calculated reflections in the range $5^{\circ}-25^{\circ}(2$ $\Theta)$ together with the indexing. Because of the low symmetry and the quite large cell volume only a limited number of calculated peaks were observed. Therefore the reliability of the indexing is restricted and should be checked by a single crystal investigation. X-ray measurements on single crystals of fraction 3 which were identified as $\alpha$ - $\mathrm{Alq}_{3}$ confirmed the triclinic unit cell given by Brinkmann and co-workers on the basis of powder data. ${ }^{14} \mathrm{~A}$ careful examination gave indications of a threefold super structure, which is still under investigation.

\section{B. Vibrational spectroscopy}

The infrared spectra were identical for the source $\mathrm{Alq}_{3}$ material, the different fractions obtained by the sublimation process in the glass tube and for amorphous films deposited by evaporation with the substrate at room temperature. The spectral position as well as the relative intensity of the bands are consistent with published infrared data. ${ }^{21}$ The Raman 


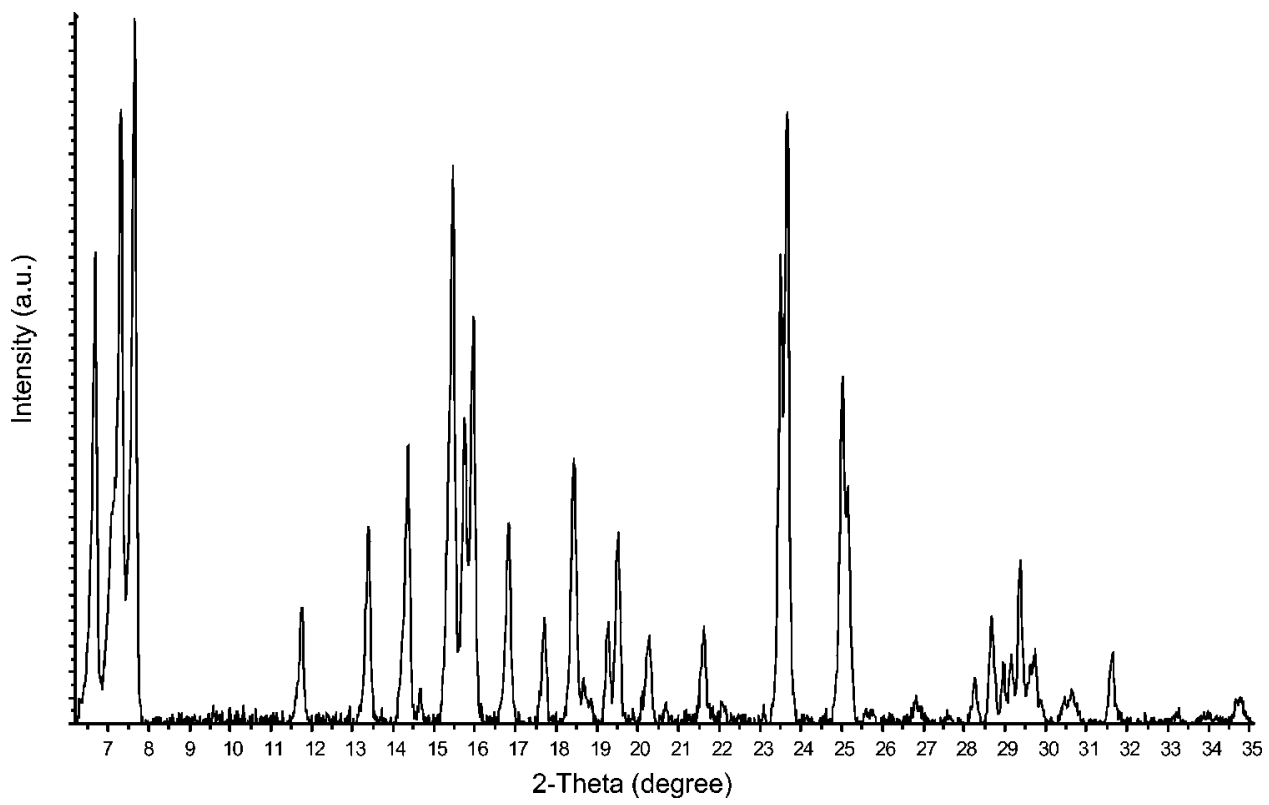

FIG. 3. X-ray powder diffraction spectrum of $\mathrm{Alq}_{3}$ fraction 1 used for additional characterization ( $8 h$, step width $\Delta 2 \Theta=0.017^{\circ}$ ). spectra of the studied materials in the spectral range 700$1700 \mathrm{~cm}^{-1}$ were also identical to the already published results. $^{14}$

Differences in the vibrational properties between fraction 1 and the other fractions appear in the Raman spectra in the spectral range $70-700 \mathrm{~cm}^{-1}$ and $2900-3200 \mathrm{~cm}^{-1}$ (Fig. 4). We were not able to find differences in the Raman spectra between fraction 2 and fraction 3 . The band positions and the

TABLE I. Observed and calculated peaks from the x-ray powder diffractogram of fraction $1\left(\delta-\mathrm{Alq}_{3}, \mathrm{Fig} .3\right)$ in the $2 \Theta$ range $5-25^{\circ}$ together with the indexing.

\begin{tabular}{crrrrcr}
\hline \hline Peak No. & $2 \Theta\left({ }^{\circ}\right)($ obs. $)$ & \multicolumn{1}{c}{$H$} & \multicolumn{1}{c}{$K$} & $L$ & $2 \Theta\left({ }^{\circ}\right)$ (calc.) & Intensity (a.u.) \\
\hline 1 & 6.650 & 1 & 0 & 0 & 6.679 & 67.7 \\
2 & 7.048 & 0 & 1 & 0 & 7.039 & 31.0 \\
3 & 7.284 & 0 & 0 & 1 & 7.269 & 87.3 \\
4 & 7.618 & -1 & 0 & 1 & 7.620 & 100.0 \\
5 & 11.727 & 1 & 0 & 1 & 11.712 & 17.3 \\
6 & 13.362 & 1 & 1 & 1 & 13.322 & 27.9 \\
& & -1 & 0 & 2 & 13.332 & \\
& & 2 & 0 & 0 & 13.381 & \\
7 & 14.335 & & & & & 40.2 \\
8 & 14.636 & & & & & 4.9 \\
9 & 15.321 & -2 & 0 & 2 & 15.273 & 36.3 \\
10 & 15.427 & & & & & 79.2 \\
11 & 15.731 & 1 & 2 & 0 & 15.724 & 43.3 \\
12 & 15.944 & & & & & 57.7 \\
13 & 16.807 & -1 & -2 & 1 & 16.832 & 28.3 \\
14 & 17.677 & 2 & 0 & 1 & 17.669 & 15.0 \\
15 & 18.411 & 1 & 0 & 2 & 18.364 & 37.4 \\
& & -3 & 0 & 1 & 18.402 & \\
16 & 18.646 & & & & & 7.2 \\
17 & 19.247 & 2 & -1 & 1 & 19.250 & 14.4 \\
18 & 19.492 & -3 & 0 & 2 & 19.483 & 27.4 \\
19 & 20.249 & 1 & -1 & 2 & 20.216 & 12.5 \\
20 & 20.664 & -1 & -2 & 2 & 20.665 & 3.0 \\
21 & 21.588 & & & & & 13.7 \\
22 & 22.067 & -2 & -2 & 2 & 22.058 & 3.1 \\
23 & 23.488 & -2 & 2 & 3 & 23.445 & 66.4 \\
24 & 23.642 & -1 & 3 & 2 & 23.640 & 87.1 \\
25 & 25.010 & -2 & 3 & 0 & 24.977 & 49.1 \\
\hline \hline
\end{tabular}

relative intensities are summarized in Table II together with an interpretation after Halls and Aroca ${ }^{22}$ which has preliminary character since the calculations in this reference were performed only for the facial isomer. The spectra in Fig. 4 were normalized to the intensity of the most intensive band at $116 \mathrm{~cm}^{-1}$. The position of the lowest energetic band at 77 $\mathrm{cm}^{-1}$ is influenced by the notch filter used in the Raman measurements. The transmission of the notch filter was measured by a white light source placed instead of a sample and is higher than $38 \%$ for Raman shifts of more than $70 \mathrm{~cm}^{-1}$. After correction for its transmission the position of the 77 $\mathrm{cm}^{-1}$ band for fraction 1 did not change whereas it shifted by about $5 \mathrm{~cm}^{-1}$ to lower energies for fraction 3 . Halls and Aroca $^{22}$ also reported the presence of a band at $71 \mathrm{~cm}^{-1}$ without giving specifications about the used notch filter. One can find bands in both spectra in Fig. 4 which are either the same or are shifted by less than $10 \mathrm{~cm}^{-1}$. However, the number of the observed modes for fraction 1 is less than for fraction 3 . It is suggestive for the presence of a higher sym-

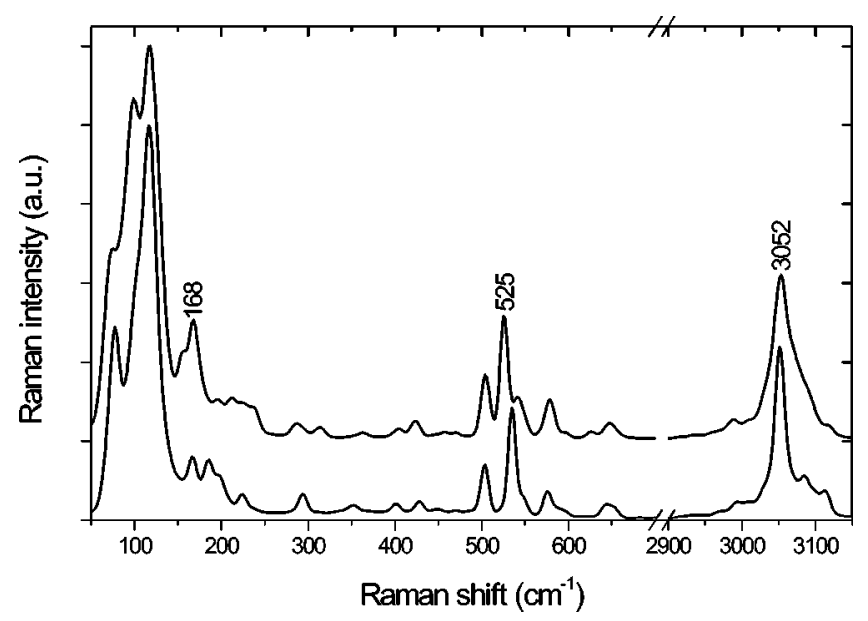

FIG. 4. Raman spectra of polycrystalline $\mathrm{Alq}_{3}$ fraction 1 (lower trace) and 3 (upper trace) taken at room temperature. 
TABLE II. Energetic position and intensity of the Raman active modes of crystalline $\mathrm{Alq}_{3}$ fractions 3 (first and second column) and 1 (third and fourth column) together with their assignment according to Ref. 22.

\begin{tabular}{|c|c|c|c|c|}
\hline $\begin{array}{l}\text { Energy } \\
\left(\mathrm{cm}^{-1}\right)\end{array}$ & $\begin{array}{l}\text { Intensity } \\
\text { (a.u.) }\end{array}$ & $\begin{array}{l}\text { Energy } \\
\left(\mathrm{cm}^{-1}\right)\end{array}$ & $\begin{array}{l}\text { Intensity } \\
\text { (a.u.) }\end{array}$ & $\begin{array}{l}\text { Assignment } \\
\text { (Ref. 22) }\end{array}$ \\
\hline 75 & 0.487 & 77 & 0.489 & Al-oxine def. \\
\hline 99 & 0.866 & 99 & 0.55 & Al-oxine def. \\
\hline 116 & 1 & 116 & 1 & Al-oxine def. \\
\hline 157 & 0.228 & & & Oxine wag \\
\hline \multirow[t]{2}{*}{168} & 0.306 & 168 & 0.157 & Oxine wag \\
\hline & & 186 & 0.152 & Ring torsion \\
\hline 195 & 0.106 & 195 & 0.115 & Oxine wag \\
\hline 213 & 0.11 & & & Oxine wag \\
\hline 224 & 0.098 & 224 & 0.066 & Oxine wag \\
\hline 236 & 0.088 & & & Ring torsion \\
\hline 287 & 0.044 & 294 & 0.066 & Ring torsion \\
\hline 313 & 0.035 & & & AlN str. + CCO bend \\
\hline 363 & 0.022 & 351 & 0.037 & Ring def. + AlOC bend \\
\hline 405 & 0.031 & 402 & 0.042 & Ring def. + AlO str. \\
\hline 423 & 0.051 & 429 & 0.047 & Ring def. \\
\hline 448 & 0.017 & 448 & 0.028 & $\mathrm{CH}$ torsion \\
\hline 457 & 0.023 & & & $\mathrm{CH}$ torsion \\
\hline 469 & 0.021 & 469 & 0.024 & Ring def. \\
\hline 504 & 0.168 & 504 & 0.14 & Ring def. \\
\hline 525 & 0.317 & 534 & 0.286 & Ring def. + $\mathrm{AlO}$ str. \\
\hline 540 & 0.111 & 548 & 0.059 & Ring def. + $\mathrm{AlO}$ str. + AlN str. \\
\hline 579 & 0.106 & 575 & 0.073 & Ring def. + AlOC bend \\
\hline 596 & 0.022 & 596 & 0.024 & $\mathrm{CH}$ torsion \\
\hline 627 & 0.024 & & & Ring def. + $\mathrm{AlO}$ str. + AlN str. \\
\hline 646 & 0.046 & 644 & 0.04 & Ring def. + AlO str. \\
\hline 2990 & 0.055 & 2993 & 0.046 & $\mathrm{CH}$ stretch \\
\hline 3052 & 0.42 & 3052 & 0.439 & $\mathrm{CH}$ stretch \\
\hline 3084 & 0.157 & 3084 & 0.112 & $\mathrm{CH}$ stretch \\
\hline 3113 & 0.042 & 3113 & 0.075 & $\mathrm{CH}$ stretch \\
\hline
\end{tabular}

metry of the crystal or molecular structure of fraction 1.

The spectrum for fraction 3 is identical to published results. ${ }^{14,22}$ The $\alpha-\mathrm{Alq}_{3}$ and $\beta$ - $\mathrm{Alq}_{3}$ crystalline phases reported in Ref. 14 have triclinic unit cells (space group P-1) and the Raman spectra are identical except for the bands in the range $150-190 \mathrm{~cm}^{-1}$. For the $\gamma$-phase a trigonal unit cell (space group P-31c) was reported, however, no Raman data were published. In our case, the analysis of the x-ray data of fractions 1 and 3, presented in the preceding section, gave triclinic unit cells for both materials. Therefore the significant differences in the Raman spectra should be ascribed to differences in the molecular symmetry rather than in the crystal symmetry.

The bands in the spectral region $2900-3200 \mathrm{~cm}^{-1}$ can be unambiguously assigned to $\mathrm{C}-\mathrm{H}$ stretching vibrations. These bands are better resolved for fraction 1 which can be either due to the presence of additional nonresolved modes or due to higher disorder in fraction 3.

\section{Photoluminescence spectra}

Figure 5 presents the low temperature photoluminescence (PL) spectra of the different fractions obtained by the sublimation process in the glass tube. There is a gradual shift of the PL maximum from $19800 \mathrm{~cm}^{-1}(2.46 \mathrm{eV})$ for fraction 3 to $21200 \mathrm{~cm}^{-1}(2.63 \mathrm{eV})$ for fraction 1 . The onset of these emissions also shifts from $2.74 \mathrm{eV}$ to $2.92 \mathrm{eV}$. Such an extremely blueshifted photoluminescence has so far not been

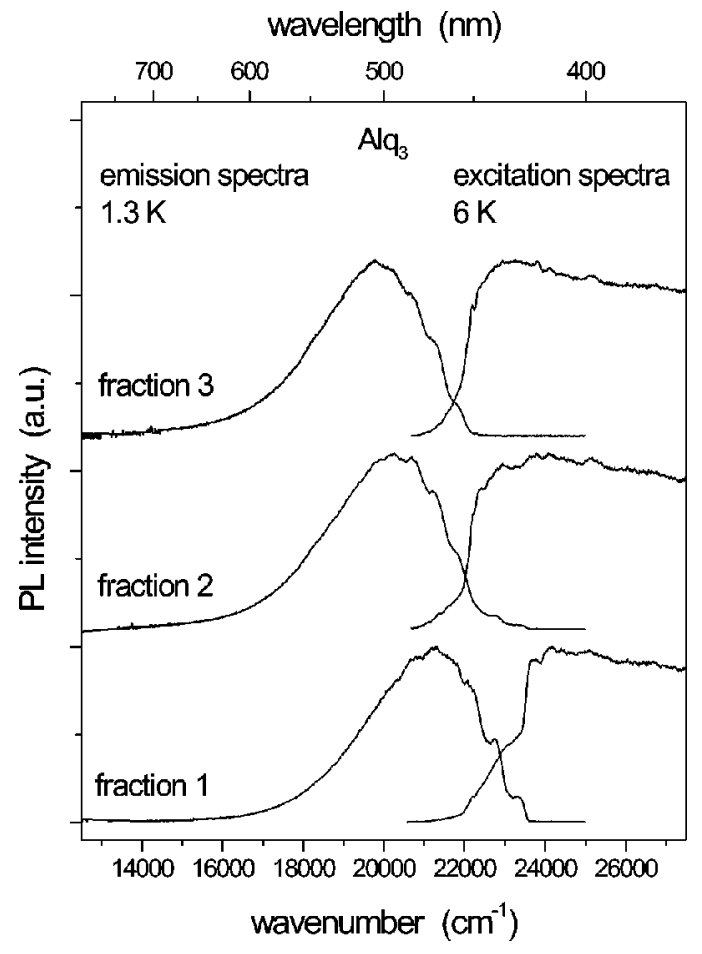

FIG. 5. Photoluminescence emission and excitation spectra of the different polycrystalline $\mathrm{Alq}_{3}$ fractions obtained by the sublimation process. The emission spectra were taken at $1.3 \mathrm{~K}$ by using excitation at $363.8 \mathrm{~nm}$. The excitation spectra were taken at $6 \mathrm{~K}$ and the detection wavelength was 500 $\mathrm{nm}$ in all cases.

reported for $\mathrm{Alq}_{3}$. All spectra consist of a broad band which is superimposed on the high energy side by a vibronic progression. Such well-resolved vibronic modes are not observed in amorphous films prepared by evaporation onto substrates at room temperature using any of these fractions as source material.

The PL excitation spectra and the site selective PL measurements presented in Figs. 5 and 6 give more insight into the nature of the fluorescence. The PL excitation spectra of fraction 1 detected at 450 and $470 \mathrm{~nm}$ in Fig. 6 show a sharp excitation edge at $23500 \mathrm{~cm}^{-1}(2.92 \mathrm{eV})$ with well-resolved and reproducible vibronic modes. The PL spectrum excited at $400 \mathrm{~nm}$ also shows vibronic progression with the $0-0$ transition at $23400 \mathrm{~cm}^{-1}(2.9 \mathrm{eV})$ and a small Stokes shift of only $200 \mathrm{~cm}^{-1}(25 \mathrm{meV})$. This vibronic progression in the PL spectrum is seen only for excitation energies above the sharp excitation edge at $2.92 \mathrm{eV}$. Upon excitation below this edge the vibronic progression in the PL disappears and the resulting spectrum is characterized by a broad band which is still blueshifted relative to the PL of the other fractions. The further decrease of the excitation energy leads to an increasing shift of the PL maximum to lower energies. Such a behavior can be described by involving carrier thermalization in lower energetic tail states similar to the case of amorphous films. ${ }^{23,24}$ The existence of states below the excitation edge is confirmed by the excitation spectra detected at 470 and 560 $\mathrm{nm}$ (Fig. 6) as well as by absorption measurements on polycrystalline samples using photothermal deflection spectroscopy (not shown). The broad feature below the excitation edge becomes more pronounced at lower detection energy 


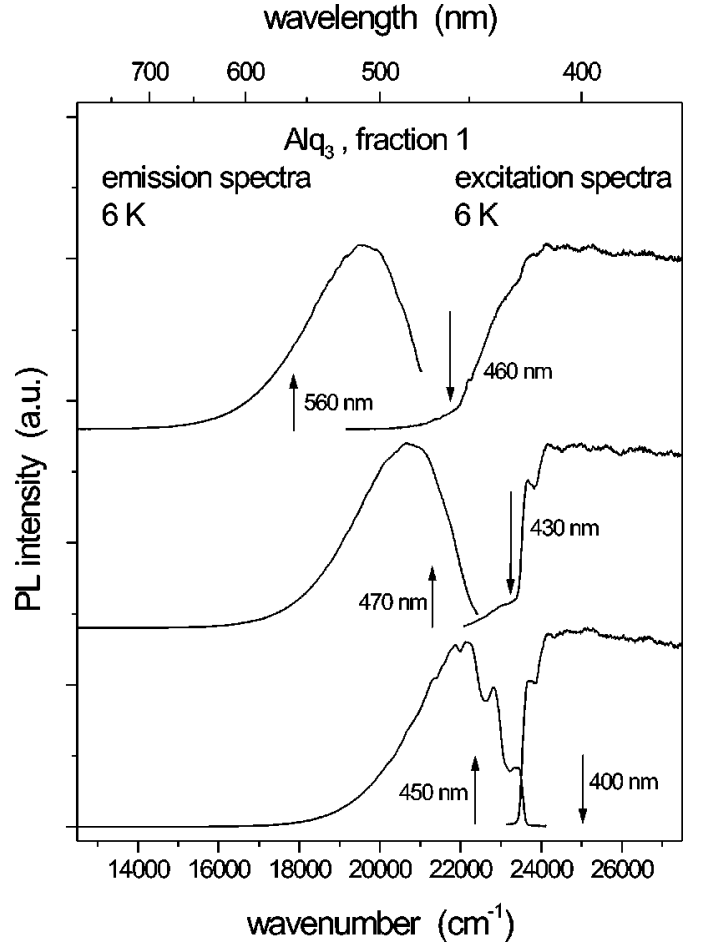

FIG. 6. Photoluminescence emission and excitation spectra of polycrystalline $\mathrm{Alq}_{3}$ fraction 1 . The measurements were performed at $6 \mathrm{~K}$. The respective detection wavelength is labeled by up arrows and the excitation wavelength by down arrows.

confirming the heterogeneous character of the photoluminescence.

Vibronic progression in the PL spectrum is also seen for fraction 3 in Fig. 5. However, the highest energetic vibronic band which corresponds to the $0-0$ transition is at 21700 $\mathrm{cm}^{-1}(2.69 \mathrm{eV})$. The energetic difference between the observed vibronic bands was determined for all fractions to about $550 \mathrm{~cm}^{-1}$. The observed shift $\left(<10 \mathrm{~cm}^{-1}\right)$ of the bands in the Raman spectra of fraction 1 and fraction 3 is below the resolution of the PL measurements. The PL spectrum of fraction 2 (Figs. 5 and 7) shows vibronic bands typical for fractions 1 and 3. However, the difference between the vibronic bands at $22850 \mathrm{~cm}^{-1}$ and $21700 \mathrm{~cm}^{-1}$ is 1150 $\mathrm{cm}^{-1}$ which indicates a different origin of the first two vibronic bands in this fraction. A confirmation for this notion comes from the excitation spectra of fraction 2 shown in Fig. 7. Detection at the high energy side $(450 \mathrm{~nm})$ of the PL band results in an excitation spectrum with an edge like for fraction 1. Detection at lower energy $(470 \mathrm{~nm})$ gives an excitation edge redshifted by $1300 \mathrm{~cm}^{-1}(0.16 \mathrm{eV})$. Further lowering of the detection energy (500 and $540 \mathrm{~nm}$ ) leads to the development of a broad feature below the excitation edge similar to the case of fraction 3. This allows to conclude that fraction 2 is constituted mainly of fraction 3 with inclusions of fraction 1 which is consistent with the above discussed $\mathrm{x}$-ray spectra. This is an important issue, because this fraction is taken very often for device fabrication. Another important conclusion is that fractions 1 and 3 are characterized by energetically displaced $0-0$ vibronic bands in addition to the displacement of the overall PL maximum.

For the investigation of the triplet state of the $\mathrm{Alq}_{3}$ frac-

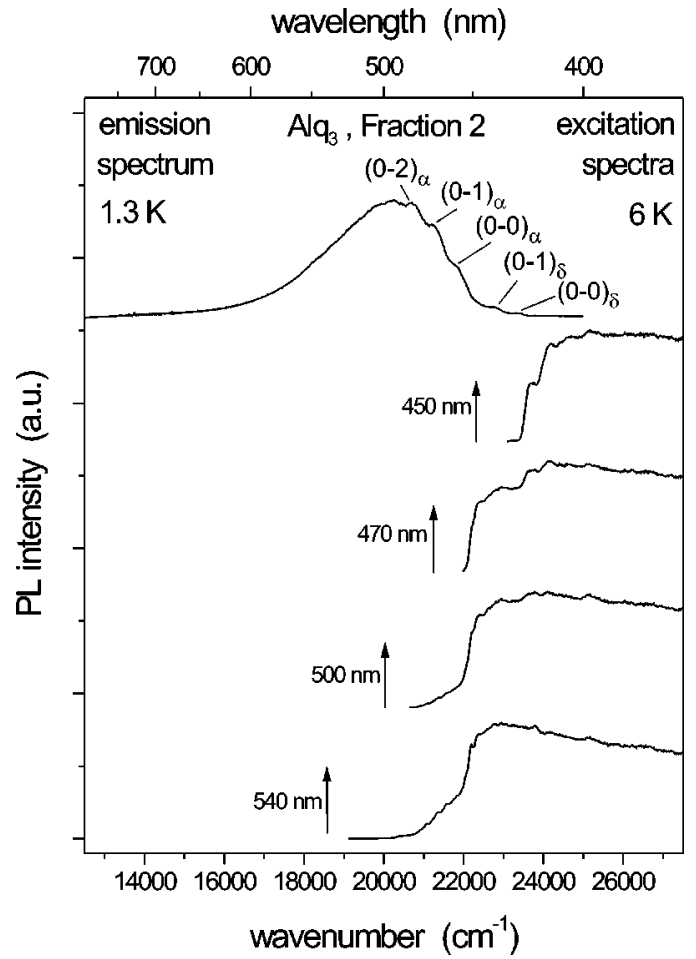

FIG. 7. Photoluminescence spectra of polycrystalline $\mathrm{Alq}_{3}$ fraction 2 . The emission spectrum was taken at $1.3 \mathrm{~K}$ by using excitation at $363.8 \mathrm{~nm}$. The excitation measurements were performed at $6 \mathrm{~K}$. The respective detection wavelength is labeled by up arrows. The different vibronic modes belonging to the $\alpha$ and $\delta$ phase are labeled for clarity.

tions we measured the delayed emission spectra and performed pulsed experiments in the ms range. The delayed emission spectra (not shown here) of all samples are nearly identical to the prompt PL spectra of Fig. 6 with only a slight rise of the red emission tail which may be due to deep traps. The overall lifetime of the delayed emission is in the range of $10 \mathrm{~ms}$.

Figure 8 shows time traces of the emission for all $\mathrm{Alq}_{3}$ fractions (detected at $2.64 \mathrm{eV}$ for fraction 1 and at $2.48 \mathrm{eV}$ for fractions 2 and 3). Instantaneous with the turning-on of the excitation light the fluorescence is observed, which subsequently decreases with a decay time of about $10 \mathrm{~ms}$ to an equilibrium value. For fractions 2 and 3 this equilibrium value is $75 \%$ of the initial fluorescence intensity, whereas for fraction 1 the intensity remains at a level of $98 \%$ of the initial value. It has to be noted that the time traces for an amorphous film (not shown here) are similar to those for fractions 2 and 3 with the same decrease to about $75 \%$ of the initial value.

The observed decrease of the fluorescence intensity is due to the increase of the percentage of molecules, which are in the long-lived triplet state and therefore not able to emit fluorescence light. This means that in fractions 2 and 3 at the given experimental conditions about $25 \%$ of the molecules are in the triplet state whereas for fraction 1 these are only $2 \%$ of the molecules.

For an estimation of the triplet lifetime we recorded the delayed emission decay and received for all fractions similar decay traces: fractions 2 and 3 [bi-exponential: $7 \mathrm{~ms}(95 \%)$, $1 \mathrm{~ms}(5 \%)$ ] and fraction 1 [bi-exponential: $5 \mathrm{~ms}$ (90\%), $1 \mathrm{~ms}$ 


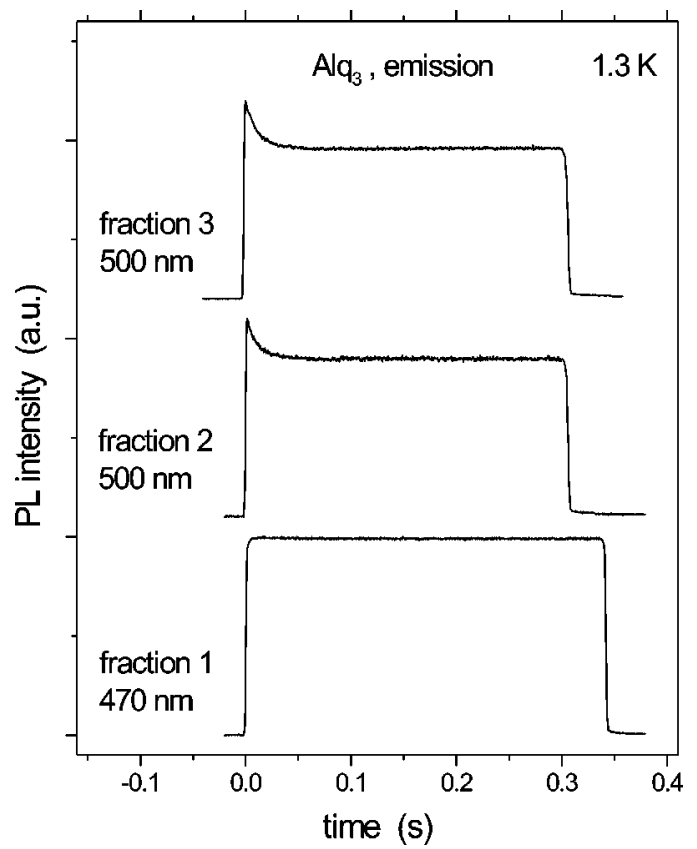

FIG. 8. The time dependence of the fluorescence in the ms-time regime during an optical excitation pulse for polycrystalline $\mathrm{Alq}_{3}$ fractions 1, 2, and 3. The measurements were performed at $1.3 \mathrm{~K}$ by using excitation at 363.8 $\mathrm{nm}$. The detection wavelength is labeled in the figure.

$(10 \%)]$. From the similarity of the triplet lifetimes we conclude that the reduced population of the triplet state for fraction 1 is due to a strongly reduced intersystem crossing process which populates the triplet state.

\section{DISCUSSION}

The experimental results in the present paper clearly show the appearance of a new crystalline phase of $\mathrm{Alq}_{3}$ so far not described in the literature. Its crystal structure is of low symmetry (triclinic) and the unit cell volume is doubled compared to $\alpha$ - $\mathrm{Alq}_{3}$. We propose a triclinic unit cell with $a=14.501(15) \AA, b=12.651(14) \AA, c=13.402(11) \AA, \alpha$ $=82.74(7)^{\circ}, \quad \beta=114.22(7)^{\circ}, \quad \gamma=93.85(16)^{\circ}, \quad$ and $V$ $=2224(2) \AA^{3}$. The enlarged cell volume with $4 \mathrm{Alq}_{3}$ complexes per unit cell may be required by the occurrence of different enantiomers. According to the markedly different properties we suppose that the new crystalline phase (hereafter $\delta$ phase) contains the facial isomer of $\mathrm{Alq}_{3}$. This would also explain the different behavior against solvents.

The differences in the Raman spectra between the two fractions 1 and 3 indicate higher symmetry for fraction 1 . This also suggests that the new phase consists of molecules in the facial isomer state, which is of higher symmetry than the meridianal one. Recently, Curioni et al. published results of ab initio calculations for the two isomers of $\mathrm{Alq}_{3} \cdot{ }^{15} \mathrm{In}$ this study they calculated the energy gap for both isomers and found that it is by $0.3 \mathrm{eV}$ larger in the facial isomer. This value is indeed comparable with the blueshift of about 0.2 $\mathrm{eV}$ observed for the maximum of the PL and the 0-0 transitions of fraction 1 relative to those of fraction 3 . It has to be noted that the energetic position of the $0-0$ vibronic band in the PL of fraction 3 is consistent with the one of the $\alpha$ phase of $\mathrm{Alq}_{3}$ reported in Ref. 14, which confirms our identification of fraction 3 with the $\alpha$ phase. The origin of the vibronic progression can be found in the coupling of the electronic transition to vibronic transitions with energies around 550 $\mathrm{cm}^{-1}$ as discussed by Brinkmann et al. for the $\alpha$ phase of $\mathrm{Alq}_{3} .{ }^{14}$ It can be seen in Table II that there are several modes around this energy which makes a unique assignment without further data not possible. The matrix isolation experiments of Kushto et al. ${ }^{21}$ have shown that vibronic progression with an average distance between the vibronic bands of about $550 \mathrm{~cm}^{-1}$ can also be detected for amorphous films if the $\mathrm{Alq}_{3}$ molecules are spatially well separated.

Our site selective PL measurements clearly show that the low temperature emission spectra of crystalline $\mathrm{Alq}_{3}$ consist of two components-a broad featureless band and a wellresolved vibronic progression. Both of them are sensitive to the molecular isomery and are blueshifted for the facial isomer. The broad band can appear because the excited carriers thermalize in the tail states of the HOMO, respectively, LUMO levels. Such tail states can be ascribed to potential fluctuations in the material similar to the case of amorphous films. They have also been detected in absorption measurements on polycrystalline samples as a characteristic exponential region below the optical gap. ${ }^{24}$ Although the x-ray analysis gave no evidence for the presence of an additional amorphous phase, small amounts of amorphous material could also result in the observed featureless emission. However, in this case one would not expect to see a shift of the spectral position of this emission feature between fraction 3 and 1.

An important point is the similarity of the population of the triplet states for amorphous films and the crystalline $\alpha$ phase of $\mathrm{Alq}_{3}$ (meridianal isomer, fraction 3 and 2 in Fig. 8). This indicates that crystallinity or disorder of the sample or the molecular packing in a crystal is of minor importance for this process. Obviously, this is a property of the molecules in the meridianal configuration. So the observation of the reduced triplet population for the $\delta$ phase of $\mathrm{Alq}_{3}$ (fraction 1 in Fig. 8) favors an intramolecular process as the explanation for the strongly reduced intersystem crossing, which would be provided by a changed molecular symmetry due to the isomery of the $\mathrm{Alq}_{3}$ molecule.

\section{CONCLUSIONS}

A new crystalline phase of unsolvated $\mathrm{Alq}_{3}\left(\delta-\mathrm{Alq}_{3}\right)$ has been obtained by sublimation. As compared to the already known phases of $\mathrm{Alq}_{3}$, it is characterized by a blueshift of the fluorescence, larger unit cell volume, specific differences in the Raman spectra and a decreased formation of triplet states. These differences are tentatively explained by the isomery of the $\mathrm{Alq}_{3}$ molecule. We suggest that the $\delta$ phase of $\mathrm{Alq}_{3}$ consists of the facial isomer. Of course this interpretation has preliminary character as long as there are no data on single crystals of the $\delta$ phase allowing a clear assignment of the positions of all atoms within the unit cell and thus a verification of the presence of the facial isomer. Therefore further efforts will aim at the optimization of the sublimation process in order to obtain larger crystals suitable for a single crystal structure analysis. 


\section{ACKNOWLEDGMENTS}

This work has been partly funded by the Deutsche Forschungsgemeinschaft. M. Tzolov would like to acknowledge the financial support of the Alexander von Humboldt Foundation.

${ }^{1}$ W. E. Ohnesorge and L. B. Rogers, Spectrochim. Acta 15, 27 (1959).

${ }^{2}$ R. A. Chalmers and M. A. Basit, Analyst (Cambridge, U.K.) 92, 680 (1967).

${ }^{3}$ C. W. Tang and S. A. VanSlyke, Appl. Phys. Lett. 51, 913 (1987).

${ }^{4}$ C. W. Tang, S. A. VanSlyke, and C. H. Chen, J. Appl. Phys. 65, 3610 (1989).

${ }^{5}$ J. Shi and C. W. Tang, Appl. Phys. Lett. 70, 1665 (1997).

${ }^{6}$ L. S. Hung, C. W. Tang, and M. G. Mason, Appl. Phys. Lett. 70, 152 (1997).

${ }^{7}$ H. Aziz, Z. D. Popovic, N.-X. Hu, A.-M. Hor, and G. Xu, Science 283, 1900 (1999).

${ }^{8}$ H. Kubota, S. Miyaguchi, S. Ishizuka et al., J. Lumin. 87-89, 56 (2000).

${ }^{9}$ T. Tsutsui, M. J. Yang, M. Yahiro, K. Nakamura, T. Watanabe, T. Tsuji,

Y. Fukuda, T. Wakimoto, and S. Miyaguchi, Jpn. J. Appl. Phys., Part 2 38, L1502 (2000).

${ }^{10}$ P. E. Burrows, Z. Shen, V. Bulovic, D. M. McCarty, S. R. Forrest, J. A.
Cronin, and M. E. Thompson, J. Appl. Phys. 79, 7991 (1996).

${ }^{11}$ S. Barth, U. Wolf, H. Bässler, P. Müller, H. Riel, H. Vestweber, P. F. Seidler, and W. Rieß, Phys. Rev. B 60, 8791 (1999).

${ }^{12}$ M. Stößel, J. Staudigel, F. Steuber, J. Blässing, J. Simmerer, and A. Winnacker, Appl. Phys. Lett. 76, 115 (2000).

${ }^{13}$ W. Brütting, S. Berleb, and A. Mückl, Org. Electron. 2, 1 (2001).

${ }^{14}$ M. Brinkmann, G. Gadret, M. Muccini, C. Taliani, N. Masciocchi, and A. Sironi, J. Am. Chem. Soc. 122, 5147 (2000).

${ }^{15}$ A. Curioni, M. Boero, and W. Andreoni, Chem. Phys. Lett. 294, 263 (1998).

${ }^{16}$ R. L. Martin, J. D. Kress, I. H. Campbell, and D. L. Smith, Phys. Rev. B 61, 15804 (2000).

${ }^{17}$ J. R. Majer and M. J. A. Reade, Chem. Commun. (London) 1970, 58 (1970).

${ }^{18}$ A. I. Vogel, Textbook of Quantitative Chemical Analysis, 5th ed. (Longman, New York, 1989).

${ }^{19}$ D. Louer and M. Louer, J. Appl. Crystallogr. 5, 271 (1972).

${ }^{20}$ A. Boultif and D. Louer, J. Appl. Crystallogr. 24, 987 (1991).

${ }^{21}$ G. Kushto, Y. Iizumi, J. Kido, and Z. Kafafi, J. Phys. Chem. A 104, 3670 (2000).

${ }^{22}$ M. D. Halls and R. Aroca, Can. J. Chem. 76, 1730 (1998).

${ }^{23}$ W. Humbs, H. Zhang, and M. Glasbeek, Chem. Phys. 254, 319 (2000).

${ }^{24}$ M. Tzolov, W. Brütting, V. Petrova-Koch, A. Mückl, S. Berleb, J. Gmeiner, and M. Schwoerer, Proc. ICSM 2000, Synth. Met. (in press). 